Perspective

\title{
The formula of the waste of cholesterol a new way to increase over-life in patients affected from breast cancer - A prospective study
}

\begin{abstract}
Introduction: Breast cancer represents the first incidence of tumors in the women in occidental countries including Cuba. In this paper we analyze it, taking into account the formula of the waste of cholesterol, and how we can see what we are doing in the adjuvant setting in a prospective and observational study that began in the year 2001.
\end{abstract}

Material and methods: According to the received treatment 365 records of patients with operable breast cancer were reviewed, and relapses before 5 years were analyzed. One group received chemotherapy alone and the other group received chemotherapy +tamoxiefen. The criterion of treatment was based on estrogen receptor status or clinical criteria of hormone dependence. Patients were staged by the TNM classification. Patients' data were collected from the clinical records from the archives of the III Congreso Hospital and were translated to a digital sheet where levels of total cholesterol and HDL cholesterol are introduced, classifying the tumors in high waste of cholesterol or low waste of cholesterol.

Results: It was found that the patients with negative waste of cholesterol that received chemotherapy alone had a significant increase of relapses in all stages, including stage I. The results were compared with the paper presented in St Gallen 2001, where the patients did not receive adjuvant therapy. It was seen that when the unbalance of cholesterols increase in patients with negative waste of cholesterol that received adjuvant chemotherapy but did not receive tamoxifen afterwards the percentage of relapses increases. We concluded that adjuvant chemotherapy has to be discussed in patients with negative waste of cholesterol and if it is used it has to be followed by treatment with tamoxifen.
Volume 5 Issue 3 - 2016

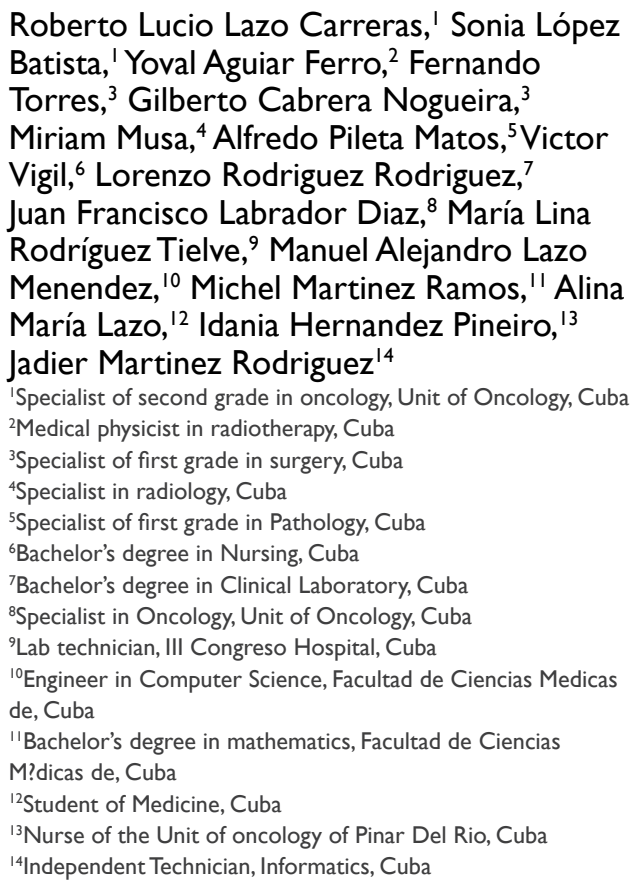

Correspondence: Roberto Lucio Lazo Carreras, Specialist of second grade in oncology, Unit of Oncology of Pinar Del Rio, Cuba,

Email lazzo@princesca.pri.sld.cu; carreraslazzo@gmail.com

Received: May 20,2016 | Published: July 20, 2016

\section{Introduction}

The waste of cholesterol formula was presented in the St Gallen Conference on Adjuvant therapy of Primary Breast Cancer in the year 2001. ${ }^{1}$ It was performed taking into account the normal levels of cholesterol in the normal female population according to age, and the relation among total cholesterol, Seri estrogens and estrogenic receptors in patients affected by breast cancer. It is a dynamic formula inside a digital sheet that allows us to know the normal levels of the waste of cholesterol for estrogen formation according to age. Normal population has to be in Zero, when the levels of total cholesterol and HDL cholesterol of patients with breast cancer are introduced in the digital sheet the cholesterol waste can be increased or decreased many times, so we can act taking into account what increases or decreases the HDL cholesterol and treat the patient to obtain the normal balance of cholesterols where the immunologic system is competent and apoptosis is available. In that paper it was found that patients of high waste of cholesterol, that are patients with increased relative levels of HDL cholesterol have the most aggressive tumors. In fact it was reported in 2012 by Chritiane Danilo that tumors with higher levels of HDL cholesterol are the most aggressive, which reinforces our results. ${ }^{2}$ On the other hand the balance of cholesterols where certain cholesterol metabolites that can promote or suppress breast cancer has been described by Sandrine Silvente-Poirot and Marc Poirot. This raises the important question of how to regulate or inhibit the cholesterol metabolic pathway, and at what step, in a therapeutic approach to cancer. ${ }^{3}$

It is known that tamoxifen increases the levels of HDL cholesterol ${ }^{4}$ and chemotherapy decreases its levels. In this paper we can see the percentage of relapse of patients in stage II affected by breast cancer, treated only with surgery and radiotherapy without adjuvant chemotherapy or hormone therapy. We could see that patients with negative waste of cholesterol less than 6 times the normal waste of cholesterol had $0 \%$ relapse before 10 years. Is the objective of this paper to know how the adjuvant treatment can be influenced in patients in relapse, taking into accounting the levels of cholesterol and the received treatment. Cholesterol is the source of steroids hormones 
including estrogens, but by this way the other steroid hormones have a participation in the balance of steroids hormones, that is affected in patients with breast cancer .In 1990 we presented a paper ${ }^{5}$ in a conference where we could see that the levels of total cholesterol are inverse to the levels of estrogens, so that that the waste of cholesterol for estrogens formation could be expressed:

\section{Waste of cholesterol $=\frac{1}{\text { Levels of Total cholesterol }}$}

Afterwards we could elaborate the formula of the steroid hormones in relation to the waste of cholesterol and the relation with HDL cholesterol as follows:

\section{Waste of cholesterol $=\frac{(\text { Thyroid action })(\text { Estrogens action })(\text { HDL cholesterol })}{(\text { Corticoids action })(\text { Progesterone action })(\text { Androgens action })(\text { LDL cholesterol })}$}

In fact this formula is the endocrine motor that has to guide breast cancer treatment and to repair the balance of cholesterol. For example, if we have a tumor of low waste that is the equivalent in our medical conception of a positive tumor estrogen receptor, we use the tamoxifen as SERMS (Selective Estrogen Receptor Modulators) but it increases HDL cholesterol as a second mechanism of action, but tamoxifen increases the levels of thyroid hormone too, so this way we are increasing the levels of HDL cholesterol and increasing the waste of cholesterol. We know estrogens are included in the risk of breast cancer; however it was used years ago before the appearing of the Selective Estrogens Receptors Modulators in advanced patients in the hormone-dependant tumors. This way hypophyseal-pituitarygonadal axis and regulation of the limbic-hypothalamic circuit can be included in this formulation and we can use drugs as Goserelin in premenopausal patients. In the other way if we use corticoids, androgens or progesterone we are decreasing the waste of cholesterol and it could be a risk of cancer just like estrogens. In the case of chemotherapy it was investigated by Chernov ${ }^{6}$ that it decreases TSH and increases ACTH, so we are decreasing the waste of cholesterol this way. So if we use chemotherapy in low waste tumors we are increasing the misbalance; but if we use tamoxifen afterwards we are replacing this balance of cholesterols again. On the other hand we can explore for its use all the elements that increase or decrease the levels of SR-B1 (the receptor of HDL cholesterol) in breast cancer treatment. ${ }^{7}$ We conducted a study that is still unpublished, ${ }^{8}$ where we can see that while the waste is farthest from Zero, there is more lymph gland metastasis and cells become more undifferentiated, both in negative waste of cholesterol and in positive waste of cholesterol.

-For now the mechanism of HDL cholesterol ${ }^{9}$ in steroidgenesis is not well known, but according to our investigation it could be involved in estrogen formation or could regulate LDL cholesterol for estrogen formation..$^{10}$ In the event of the formation of other steroid hormones (corticoids androgens and progesterone) it is directed related with LDL cholesterol. In connection with feeding and breast cancer there is not a real mechanism of action, we know that the occidental style of life and excess of intake of saturated fat lower the waste of cholesterol due to the increase of LDL cholesterol. Alcohol increases HDL cholesterol so it is involved in tumors of high waste of cholesterol, and physical exercise increases the waste of cholesterol. As for acid diet and alkaline diet, the activation or deactivation of somatostatin in the digestive tracts is described, ${ }^{11-13}$ it increases itself the thyroid gland activity and the production of thyroid hormones, so a continuous acid or alkaline diet could influence the misbalance of the waste of cholesterol. On the other hand a regression of breast cancer has been described by Dr Di Bella G with somatostatin treatment.

Vitamins use and abuse. It has been described than vitamin E low SR-BI what could be investigated in its use in the treatment of high waste tumors. B1Vitamin is essential for estrogens formation what could be use in low waste tumors as c vitamin. The low levels of D Vitamin have been described as a risk of breast cancer in fact it is built from cholesterol. The use of multivitamins could be a risk of misbalance in the system of the waste of cholesterol, because it is not of natural foods where our organism takes what he needs. The use of Antidepressants and other drugs for long time can change the waste of cholesterol because of its influence on the endocrine system; it could influence tumors of low waste of cholesterol. The stress and depressions can have an influence on endocrine disorders by increasing corticoids, so it can have an influence on the waste of cholesterol too. In graphic 1, we can see the connection of the waste of cholesterol with the brain activity, the endocrine system and cell interaction.

\section{Material and methods}

\section{Study design}

Records of 365 operable breast cancer patients treated at the Unit of Oncology of Pinar del Rio were analyzed in a prospective and observational study that began in the year 2001. Before operation patients were classified according to the stage in a multidisciplinary medical consultation where oncologist and surgeons decide treatment, and after the operation, postsurgical TNM classification was applied. Patients were operated on by modified radical mastectomy or conserving breast surgery. After operation and before starting systemic treatment total cholesterol and HDL cholesterol was indicated together with the other blood tests. They were included in the digital sheet that takes into account the formula of the waste of cholesterol. Patients were reclassified in stages according to the TNM stratification system. ${ }^{14}$ The follow-up of the patient was at least 5 years. Patients that did not relapse but did not come to medical consultation in 5 years were excluded. The used treatment of chemotherapy regime was AC 4 cycles + CMF 4 cycles or CMF. Radiotherapy was used in breast conserving surgery according to the clinical stage and other criteria stated in the consensus of St Gallen. Tamoxifen for 5 years was indicated, taking into account the estrogen receptors status, or clinical factors of hormone-dependence. ${ }^{15-17}$ Relapse or no relapse of this group of patients was compared in connection with the times the waste of cholesterol was increased or decreased and the used treatment in an observational study, and afterwards it was compared with the sample of patients presented in the St Gallen Conference in 2001, where patients were treated only with surgery (radical mastectomy + radiotherapy in stage II patients (At that time TNM were different from the present ones, but they were patients with tumors higher than $2 \mathrm{cms}$ and with or without lymph node metastasis and much of these patients could be classified as Stage III with the actual TNM classification). For the measure of total cholesterol and HDL Cholesterol it was used the enzymatic test and results were expressed in $\mathrm{mmol} / \mathrm{l}{ }^{18}$

\section{Statistical analysis}

The statistical hypothesis about the difference between the percentages of relapses in both groups of treatment was tested using chi-square test, what is equivalent to test the relationship between the 
treatments and relapses. A P-value of less than 0,001 was considered a significant difference. In addition, the Relative Risk (RR) was estimated as a measurement of the relationship mentioned above, through statistic as well as the corresponding 99, 9\% confidence interval (CI). Statistical analyses were performed using MS Excel and the Statistical Package for Social Sciences (SPSS) software tools.

This investigation was approved by the Committee of Ethics for the investigation of the III Congreso Hospital. And it fulfills the ethical principles of the letter of Helsinki for the investigation in humans.

\section{Results}

In our sample 196 patients (53.6\%) were classified as high waste of cholesterol tumors and $169(46.3 \%)$ low waste of cholesterol tumors. 130 patients $(35,6 \%)$ were classified as Stage I, 142 patients $(38,9 \%)$ as Stage II and 93 patients $(25,4 \%)$ as Stage III. Table 1 shows the different stages of all the sample of patients.

\section{Study of the present sample of patients}

Study of tumors with low waste of cholesterol: Patients of low waste were analyzed by stages in table II according to relapse before 5 years. As we can see in patients Stage I that received treatment with chemotherapy + Tamoxifen or tamoxifen alone the percent of relapse before 5 years was only $8,6 \%$. The three patients that relapsed were Stage Ic. Patients that relapsed were: one with brain metastasis, one with lymph node metastatic upper clavicle and the other with tumor in the other breast.

On the other hand, we can see the percentage of patients of Stage I, that only received chemotherapy, where the percentage of relapse increased to $64 \%$. In this group 2 patients presented local relapse, and the others lung and bone metastasis. One patient presented ovarian cancer. It is important to know that in this group two patients that did not relapse were taking policosanol, a medication that has the same movement that tamoxifen in connection with the increasing of HDL cholesterol, and other patients that did not relapse had a chemical castration with chemotherapy.

When we analyzed stage II patients, something similar is seen. Patients that received chemotherapy + Tamoxifen or Tamoxifen alone had $16,2 \%$ of relapse before 5 years. 5 patients presented local relapse, and one bone metastasis; one patient presented lung cancer. Patients that relapsed were 5 on stages IIbp and 2 on stage IIap. On the other hand patients StageII that received only chemotherapy had $66,7 \%$ of relapses before 5 years. 7 had local relapse and 5 bone metastasis and one brain and lung metastasis. Patients that relapse were 10 Stage IIbp and 3 Stage IIap.

Something interesting is seen in Stage III too. Patients that received chemotherapy + Tamoxifen or tamoxifen had $27 \%$ of relapse before 5 years. Patients that relapse were 5 Stage IIIbp and 2 Stage IIIap. Patients had 2 local relapses and the others lung, bone and liver metastasis. On the other hand patients that received chemotherapy alone had $100 \%$ of relapse before 5 years. Patients were classified 6 on stage IIIap and 7 on stage IIIcp. Four patients had local relapse and 9 had bone and visceral metastasis. When patients with negative waste of cholesterol were analyzed statistically the percentage of relapses was significant in relations with patient that received

Table I Staging system in our sample of patients adjuvant tamoxifen and the other group of patients that only received chemotherapy $(\mathrm{p}<0.001)$.

Statistical analysis: Patients with low waste of cholesterol were processed sadistically according with the received treatment by Stages. D.F. $=1$ (degrees of freedom in chi-squarer TEST, FOR ALL stages).

\section{High waste of cholesterol tumors study}

The percentage of relapses in patients with positive waste of cholesterol can be seen in table III.As we can see; there is no significant percentage of relapses according to the used treatment. It will be later examined relative to the St. Gallen Conference paper in 2001.

Comparative Study: Next we decided to study the same parameters that we analyzed in 2001 at the St Gallen conference. That way we analyzed the same four groups of parameters that we used at that time: group I patients that had an increased waste of cholesterol more than 21 times the normal waste, group II who had the waste from 1 to 20 times, group 3 who had decreased the waste of cholesterol to 5 times the normal waste, and group IV who had a waste decreased more than 5 times the normal waste of cholesterol.

Comparative Study in patients with positive waste of cholesterol: Sample of patients were compared with the sample of patients presented in St Gallen 2001; group I were the patients that had the waste of cholesterol more than 21 times the normal waste of cholesterol, and group II were the patients that had increased from 1 to 20 times the waste of cholesterol. That way we analyzed too the group of patients of the present paper. As we can see in Table \#IV, receiving adjuvant chemotherapy in tumors of high waste of cholesterol decreases the percentage of relapses in group I and II in the stage I and II but not in Stage IIII.

Comparative study in patients with negative waste of cholesterol: As we can see in Table \#V and \#VI patients of group III (waste of cholesterol decreased to 5 times the normal waste)that received chemotherapy + tamoxifen had percent of relapses for stage I ,II and III, respectively of $\mathbf{1 5 , 3} \%, \mathbf{2 8 , 5} \%$ and $\mathbf{2 3 , 8 \%}$, and patients of group IV (patients that had decreased less than 6 times the waste of cholesterol)that received chemotherapy +tamoxifen had a percentage of relapses for stage I, II and III respectively of $0 \%, 30 \%$ and 37,5 $\%$.In the group of St Gallen, in stage II patients the percentage of relapses was $9,6 \%$ for group III and $0 \%$ for group IV. Afterwards we analyzed patients of group III and IV that only received chemotherapy and we can see in group III the percentage of relapses for stage I, II and III was respectively $66 \%, 70 \%$, and $100 \%$. When we analyzed group IV the percentage of relapses by stages was $100 \%, 60 \%$ and $100 \%$.

\section{Adverse reactions to tamoxifen}

As for adverse reactions to Tamoxifen, one was seen in the group of low waste tumors that received tamoxifen. It was a tromboembolic event; on the other hand patients with positive waste of cholesterol had the other adverse reactions observed in this group of patients. In this group of patients 10 patients had to give up tamoxifen, one because of endometrial adenocarcinoma, 2 vaginal bleeding, 2 tromboembolic events, 1 thrombocytopenia, and the others because other lower effects of tamoxifen.

\begin{tabular}{|c|c|c|c|c|}
\hline Stage I $(R R=7,4$; & CI 99, 9\% LOWER: I, 7 & UPPER: 32,7 & $X 2=19,5$ & $P=0,000009)$ \\
\hline Stage II $(R R=3,7$; & $\mathrm{Cl} 99,9 \%$ LOWER I, 13 & UPPER:I2,2 & $X 2=13,2 ;$ & $P=0,0002$ \\
\hline Stage III (RR = 3, 7; & $\mathrm{Cl} 99,9 \%$ LOWER: I, 4 & UPPER: 9,6 & $X 2=19,8$ & $P=0,000008$ \\
\hline
\end{tabular}


Table continued..

\begin{tabular}{|c|c|c|c|c|c|c|}
\hline Stage & $-\mathrm{CT}$ & $-C$ & $+C$ & $+\mathrm{CT}$ & Total & $\%$ \\
\hline la & I & I & 4 & 3 & 9 & $2,4 \%$ \\
\hline lb & 5 & I & 3 & 4 & 13 & $3,5 \%$ \\
\hline Ic & 40 & 12 & 35 & 21 & 108 & $29,5 \%$ \\
\hline Ila & 28 & 14 & 12 & 13 & 67 & $18 \%$ \\
\hline Ilb & 13 & 8 & 36 & 18 & 75 & $20,5 \%$ \\
\hline Illa & 14 & 6 & 17 & 7 & 44 & $12 \%$ \\
\hline IIlb & I & 4 & 5 & 2 & 12 & $3,2 \%$ \\
\hline IIIC & 17 & 5 & 7 & 8 & 37 & $10 \%$ \\
\hline Total & 119 & 51 & 119 & 76 & 365 & $100 \%$ \\
\hline
\end{tabular}

$\mathrm{CT}$, patients with negative waste of cholesterol that receive chemotherapy + tamoxifen for 5 years or tamoxifen alone; C, patients with negative waste of cholesterol that receive only chemotherapy; $+C$, patients with positive waste of cholesterol that received only chemotherapy; $C T$, patient with positive waste of cholesterol that receives chemotherapy + tamoxifen or tamoxifen alone

Table 2 Patients with negative waste of cholesterol that show the percentage of relapses before 5 years according to the adjuvant treatment in different Stages $\mathrm{p}<0.001$

\begin{tabular}{|c|c|c|c|c|c|c|c|c|c|}
\hline & Stage I & & & Stage II & & & Sta & & \\
\hline Treatment & $\mathrm{N}$ & N Rs & $\%$ Rs & $\mathrm{N}$ & N Rs & $\%$ Rs & $\mathrm{N}$ & N Rs & $\%$ Rs \\
\hline Chemo + TMX or TMX alone & 46 & 4 & $8,6 \%$ & 42 & 7 & $16,6 \%$ & 33 & 9 & 27,2 \\
\hline Chemotherapy & 14 & 9 & $64 \%$ & 21 & 13 & $61,9 \%$ & 13 & 13 & $100 \%$ \\
\hline Total & 60 & 13 & $21,6 \%$ & 63 & 20 & $31,7 \%$ & 46 & 22 & $47,8 \%$ \\
\hline
\end{tabular}

Table 3 Shows the percentage of relapses according to the adjuvant treatment in different stages in patients with positive waste of cholesterol

\begin{tabular}{|c|c|c|c|c|c|c|c|c|c|}
\hline \multirow[b]{2}{*}{ Treatment } & \multicolumn{3}{|c|}{ Stage I } & \multicolumn{3}{|c|}{ Stage II } & \multicolumn{2}{|c|}{ Stage III } & \multirow[b]{2}{*}{$\%$ Rs } \\
\hline & $\mathrm{N}$ & N Rs & $\%$ Rs & $\mathrm{N}$ & N Rs & $\%$ Rs & $\mathrm{N}$ & N Rs & \\
\hline Chemo + TMX or TMX alone & 29 & 2 & $6,8 \%$ & 32 & 4 & $12,5 \%$ & 17 & 6 & $35,2 \%$ \\
\hline Chemotherapy & 42 & 9 & $21,4 \%$ & 47 & 12 & $25 \%$ & 29 & 18 & $62 \%$ \\
\hline Total & 71 & II & $15,4 \%$ & 79 & 16 & $20,2 \%$ & 46 & 24 & $43,4 \%$ \\
\hline
\end{tabular}

Table 4 Comparison between the percentage of relapses in positive waste of cholesterol according to the increased waste of cholesterol in patients that did not receive adjuvant chemotherapy (presentation of St Gallen 200I) and the present paper of patients that received surgery, chemotherapy and radiotherapy in Stages I, II and III in patients with positive waste of cholesterol. SCR (Surgery + Chemotherapy + Radiotherapy)

\begin{tabular}{lllll}
\hline Groups & Stage II St Gallen 200 I & Stage I & Stage II & Stage III \\
\hline \multirow{2}{*}{ IWASTE 22 I times } & Surgery +RT & SCR & SCR & SCR \\
\cline { 2 - 5 } II $100 \%$ of $R<5$ years & $14,2 \% R$ & $25 \% R$ & $I 00 \% R$ \\
\hline
\end{tabular}

Table 5 Comparison between the percentage of relapses before 5 years in negative waste of cholesterol according to the decreased waste of cholesterol in patients that did not receive adjuvant chemotherapy (presentation of St Gallen 200I) and the present paper of patients that received surgery, chemotherapy and radiotherapy in Stages I, II and III in patients with negative waste of cholesterol. SCTR (Surgery + Chemotherapy + Tamoxifen + Radiotherapy)

\begin{tabular}{lllll}
\hline Groups & Stage II St Gallen 200I & Stage I & Stage & Stage III \\
\hline & Surgery +RT & SCTR & IISCTR & SCTR \\
\cline { 2 - 4 } III WASTE 0-5 times & $9,6 \%$ of R $<5$ years & $15,3 \% R$ & $28,5 \% R$ & $23,8 \% R$ \\
IVWASTE $<-6$ times & $0 \%$ of R<5 years & $0 \%$ R & $30 \% R$ & $37,5 \% R$ \\
\hline
\end{tabular}

Table 6 Comparison between the percentage of relapses according to the decreased waste of cholesterol (from 0 to 5 times decreased the normal waste and less than -6 times the normal waste of cholesterol) in patients that did not receive adjuvant chemotherapy (presentation of St Gallen $200 \mathrm{I}$ ) and the present paper of patients that received surgery, chemotherapy and radiotherapy in Stages I, II and III in patients with negative waste of cholesterol. SQR (Surgery + Chemotherapy + Radiotherapy)

\begin{tabular}{lllll}
\hline Groups & Stage II St Gallen 200 I & Stage I & Stage & Stage III \\
\hline & Surgery +RT & SCR & IISCR & SCR \\
\hline III WASTE 0-5 times & $9,6 \%$ of R $<5$ years & $66,6 \% R$ & $70 \% R$ & $I 00 \% R$ \\
IVWASTE $<-6$ times & $0 \%$ of R<5 years & $100 \% R$ & $60 \% R$ & $I 00 \%$ R \\
\hline
\end{tabular}

\section{Discussion}

As we can see in our results, if we see the results of St Gallen 2001 and the present study, adjuvant chemotherapy has a relevant role in decreasing the percentage of relapses in the positive waste of cholesterol patients; although in Stage III we have to consider new strategies of treatment, I think the close follow-up of these patients using the digital sheet would be one, and when the waste of cholesterol increases, it means there is an unbalance related to the undifferentiation of the cells and disease progression and we could use progressive chemotherapy trying to get the balance of cholesterols . On the other hand it looks like that adjuvant chemotherapy does not play the best role in patients with negative waste of cholesterol when it is used isolated, but it is important to specify that in the group of patients receiving chemotherapy+tamoxifen the percentage of 
relapses was lesser than with tamoxifen alone, but the sample was not high enough to analyze this group by itself. We see that in patients that received chemotherapy and afterwards did not receive tamoxifen the percentage of relapses is really significant $(p<0.001)$. When we analyzed the negative waste of cholesterol presented in the St Gallen conference, and patients with a waste of cholesterol under 6 times only treated with surgery and radiotherapy had $0 \%$ of relapses before 10 years and we see these results, patients that received chemotherapy + tamoxifen or with chemotherapy alone, where relapses increase, we think that it is time to analyze and to check our results. How can we explain these findings?

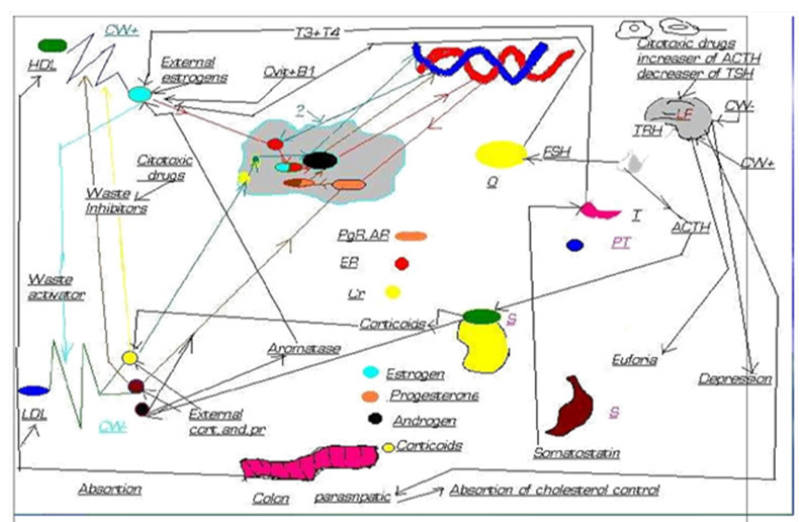

Figure I Interconnection of the waste of cholesterol with cells, digestive tracts, brain and endocrine system.

We think, as all these papers show, that there is an unbalance of cholesterols in breast cancer, there is also a connection with steroidgenesis; until now, estrogens have been the only steroid hormone related to breast cancer, and all investigations have been related to the endocrine axis, applied to the hormone dependant tumors, but in this case most of the breast cancers have a connection with all the steroids hormones, so that the indiscriminating use of corticoids as progesterone or androgens can develop an unbalance in the waste of cholesterol. We have seen in the clinical practice the use of corticoids that develop a tumor of low waste that is proven afterwards as RE+, on the other hand we have seen tumors of low waste of cholesterol that have a relapse when a high dose of corticoids have been used in other health centers because of some disease as asthma etc. In the last years the use of estrogens, androgens and progesterone has declined because of the danger that they represent, because of the medical education of the people, but still corticoids are not considered as a risk factor of breast cancer. The discovering of the receptor of SR-BI has opened a new way of treatment of breast cancer, because some investigations have been performed in cardiovascular disease that could be used in order to maintain the balance of cholesterols and therefore to control the metabolic balance of the body. It is important too, that with the use of the digital sheet of the waste of cholesterol we as medicals doctors may do dietary interventions in the follow-up of the patients. As we know, alcoholism is a risk for breast cancer in women, it increases HDL cholesterol and is a cause of tumor of high waste, those are the most aggressive tumors, and on the other hand we can recommend tamoxifen for low waste tumors and for chemoprevention of patients with low waste of cholesterol. Increase the intake of unsaturated fats, and other drugs that can lower LDL cholesterol if tamoxifen cannot maintain the balance by itself. I think that the follow-up of the patient is essential to keep the balance of the patient. New strategies of treatment should be tested for stadium III with positive waste of cholesterol. Could long-time chemotherapy for the treatment of this group in stage III be an option? Undoubtedly the close follow- up of this group of patients with the digital sheet of the waste of cholesterol could be an option, in fact we have used it in metastatic breast cancer in the tumors of high waste, and when patients relapse they do it in a waste of cholesterol higher than the initial measure before starting the systemic treatment. Patients that have a waste of cholesterol so high are generally young patients who have a big elasticity of the waste because their endocrine function is increased; old patients have an elasticity that decreases with age, it is for that reason that these patients have a long time of response to tamoxifen, including metastatic ones. We want to consider radiotherapy in this paper briefly, as we could see there were a great quantity of local relapses in the waste negative tumors, in this group of patients we usually used CMF together with radiotherapy after adriamicin course of chemotherapy. We know that a clear concept of radiotherapy is that the well differentiated tumors are more radio-resistant but more radiocurable, and undifferentiated tumors are more sensible to radiotherapy but less radio curable; that is why we consider that in the waste negative patients radiotherapy does not have to be applied together with chemotherapy including CMF schedule.

Study Limitations. Because an immune-hystochemical analysis of the tumor couldn't be performed on all patients, no comparison could be made between the waste of cholesterol with estrogens receptors and the others predictive of prognostic factors as HR3 and Ki 67 in order to show a strong connection. Nevertheless, a $50 \%$ correlation was found among negative waste of cholesterol and estrogen receptors positive and between positive waste of cholesterol and estrogen receptors negative.

As we can see, these results represent with its application a step of advance to increase survival in patients affected by breast cancer, which must be brought together with the other prognostic and predictive factors in the treatment of breast cancer. Patients of low waste of cholesterol and RE-should receive tamoxifen independently of estrogen receptor status.

\section{Acknowledgments}

We recognize the work carried out by the department of Pathology of the hospitals Abel Santamaria and the hospital Leon Cuervo Rubio, and the departments of Surgery and Clinical Laboratories of the Leon Cuervo Rubio Hospital and the contribution to this investigation.

\section{Author's contribution}

He created the Theory of the waste of cholesterol, the formula of the waste of cholesterol with the endocrine elasticity. He performed the study design. He organized the investigation, wrote the articles and review literature. He participated in the multidisciplinary Medical consultation for staging the patients and the follow-up of the patients. He participated in the prescription of chemotherapy and radiotherapy to the patients.

Sonia López Batista. Specialist of first grade in Oncology. She participated in all the investigations related before the presentation of these articles and she participated in the organization and supervision of the project of investigation. She is actually retired from the unit of Oncology of Pinar Del Rio.

Yoval Aguiar Ferro. Medical physicist in radiotherapy. $\mathrm{He}$ performed the digital sheet for the introduction of the formula of the waste of cholesterol.

Dr. Fernando Torres. Specialist of first grade in surgery. He participated in the multidisciplinary Medical consultation for staging 
the patients and the follow-up of the patients. He participated in breast cancer surgery on the patients.

Gilberto Cabrera Nogueira. Specialist of second grade in Surgery. He participated in the multidisciplinary Medical consultation for staging the patients and the follow-up of the patients. He participated in breast cancer surgery to the patients.

Miriam Musa. Specialist in radiology. She participated in the staging of the patients and in the radiologic diagnosis of relapses.

Dr Alfredo Pileta Matos. Specialist of first grade in Pathology. Assistant Professor. He participated in the staging of the patients and the histologist diagnosis

Victor Vigil. Bachelor's degree in Nursing. He participated in the administration of Chemotherapy to the patients.

Lorenzo Rodriguez Rodriguez. Bachelor's degree in Clinical Laboratory. He participated and organized the test of total cholesterol and HDL cholesterol for all the patients. Juan Francisco Labrador Diaz. Specialist in Oncology. He participated in the follow-up of the patients and in the prescription of chemotherapy and radiotherapy.

María Lina Rodríguez Tielve. Lab technician. She performed cholesterol analysis and in the organization of the investigation.

Manuel Alejandro Lazo Menendez. Engineer in Computer Science. He contributed to get references of Interest and in the revision of the manuscript.

Michel Martinez Ramos. Bachelor's degree in mathematics. He contributed to the statistical analysis of the manuscript.

Alina María Lazo. She participated in the format of tables, organization of the paper and helped in the collection of data. Student of Medicine.

Idania Hernandez Pineiro. Nurse of the Unit of oncology of Pinar Del Rio. She participated in the quest of clinical records.

Jadier Martinez Rodriguez. Independent Technician in Informatics. He performed the digital sheet where can be seen the normal elasticity for its use in chemoprevention and in cancer treatment.

\section{Conflicts of interest}

Authors declare there is not conflict of interest. All the authors agreeing the paper should be published. Database. Records of clinical histories are available in the department of Archives and statistics of the Hospital III Congreso. Calle 2da Reparto 10 de Octubre Pinar Del Rio, Cuba.

\section{References}

1. R Lazo, Lopez S, Vigil C, et al. How many times is cholesterol waste increased or decreased at time of breast cancer diagnosis? Its importance in breast cancer treatment (advanced or adjuvant therapy). The Breast 2001;10(Supp11):16
2. Danilo C. Impact of Cholesterol and Lipoproteins on Breast Cancer. ETD Collection for Thomas Jefferson University. 2012.

3. Silvente-Poirot S, Poirot M. Cholesterol and Cancer, in the balance. Science. 2014;s343(6178):1445-1446.

4. Vrbanec D, Reinier Z, Pezerovic DZ. The effect of adjuvant tamoxifen on serum lipid and lipoprotein levels. Adjuvant Therapy of primary Breast Cancer. $4^{\text {th }}$ International Conference. St Gallen/ Switzerland. 1992.

5. Carreras RL, Batista LS. Influencia del estado fisiológico en el proceso de metastización en las pacientes pre menopáusicas con cáncer de mama. Unidad Oncológica de pinar del rio Cuba, Oncología 1999;22:59.

6. Chernov VA Acción indirecta, a través del sistema endocrino, de las sustancias alquilantes en el proceso tumoral."Materiales de la primera Conferencia de quimioterapia en el cancer, en la URSS, Riga (en idioma Ruso),1968 .Tomado de el libro "La quimioterapia en las enfermedades malignas".Dr Lorenzo Alfonso Hernandez. Editorial Científico Técnica. La Habana1976 pág. 235-236

7. Loison C, Mendy F, Serougne C, et al. Dietary myristic acid modifies the HDL-cholesterol concentration and liver scavenger receptor B1 expression in the hamster. Br J Nutr. 2002;87(3):199-210.

8. Walter L Miller, Richard J Auchus. Mechanism of the steroidogenesis. The Molecular Biology, Biochemistry, and Physiology of Human steroidogenesis and its disorders. Endocr Rev. 2011;32(4):579.

9. Lazo R, Lopez S. Relationship among the HDL cholesterol, the degree of differentiation of tumor cells and in the metastatic lymph nodes in breast cancer. 2000

10. Receptor $\mathrm{Sr}-\mathrm{Bi}$ Role of type 9SR-B-I) class B scavenger receptor in HDL metabolism and atherosclerosis. A arigotti, departamento de gastroenterologia.Facultaddemedicina, Pontificia Universidadcatolica. Santiago de Chile.) Index Medicus/Medline,scorpus,IBCS,IME,MEDES Metricas sCimago journal rank(sjr);0,12.source Normalized impact per paper (SNIP):0,13.vol.num.4.julio 2000.

11. Herrera E. Somatostatina. Journal of Pharm Exp Therapy. 1999;278(2):766-773.

12. de Herder WW, Hofland LJ, Jvan der Lely A, et al. Somatostatin receptors in gastroentero-pancreatic neuroendocrine tumors. Endocrine-related Cancer. 2003;9(10):451-458.

13. Di Bella G. Complite objective response to biological therapy of plurifocal breast carcinoma. Neuro endocrinol Lett. 2008;29(6):857-866.

14. Edge SB, Byrd DR, Compton CC, et al. Breast. AJCC Cancer Staging Manual, 7th edn, New York, USA, 2010. pp. 347-376.

15. Lazo R, López S, Amador R, et al. Niveles de Colesterol Sérico en relación con los criterios Clínicos de Hormono dependencia en el Cáncer de Mama. Unidad Oncológica De Pinar del Rio Rev Cub Oncología. 1991;7(1):24-29.

16. Lazo R, Leticia F, Lage A. Variables Clínicas de pronostico individual en el cáncer mamario. Mayo-Junio. 1983;22:230-241.

17. Pascual MR, Lazo R, Lage A, et al. Clinical Factors Related to the presence of Estrogen Receptors in Breast Cancer: Prognostic stratification Analysis. Neoplasma. 1982;29(4):453-461.

18. Young DS. Effects of disease on Clinical Lab test. 4th edn, AACC. 2001. 